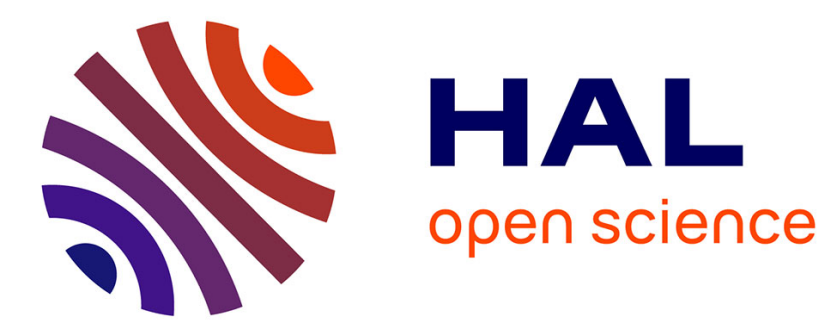

\title{
Microanalyse et microscopie électronique
}

J. Philibert

\section{- To cite this version:}

J. Philibert. Microanalyse et microscopie électronique. Revue de Physique Appliquée, 1968, 3 (4), pp.343-350. 10.1051/rphysap:0196800304034300 . jpa-00242870

\section{HAL Id: jpa-00242870 https://hal.science/jpa-00242870}

Submitted on 1 Jan 1968

HAL is a multi-disciplinary open access archive for the deposit and dissemination of scientific research documents, whether they are published or not. The documents may come from teaching and research institutions in France or abroad, or from public or private research centers.
L'archive ouverte pluridisciplinaire HAL, est destinée au dépôt et à la diffusion de documents scientifiques de niveau recherche, publiés ou non, émanant des établissements d'enseignement et de recherche français ou étrangers, des laboratoires publics ou privés. 


\title{
MIGROANALYSE ET MIGROSGOPIE ÉLEGTRONIQUE
}

\author{
Par J. PHILIBERT, \\ Institut de Recherches de la Sidérurgie Française, Saint-Germain-en-Laye.
}

\begin{abstract}
Résumé. - L'analyse chimique très locale d'un échantillon exige l'observation simultanée de celui-ci à un grandissement suffisant. A l'échelle de la microscopie électronique, deux méthodes ont été appliquées :

- La spectroscopie des électrons transmis par l'objet (lame mince) ; les pertes d'énergie de ces électrons forment en effet un spectre de raies caractéristique de l'élément chimique ou du composé traversé. Dans ce but, un filtre de vitesse doit être adjoint à un microscope électronique classique.

- La microanalyse par émission X. L'appareil est alors une combinaison de microscope électronique et de microanalyseur à sonde électronique.

Le pouvoir de résolution dans le premier cas peut être aussi bon que celui du microscope électronique ; dans le second cas, il est actuellement voisin de 2000 ou $3000 \AA$ et pourrait atteindre $200 \AA$, mais les possibilités analytiques de la microanalyse $\mathrm{X}$ sont très supérieures, car les spectres $\mathrm{X}$ sont plus fins et plus sélectifs que les spectres de pertes d'énergie. Pour les applications métallurgiques, les objets examinés peuvent être des lames minces ou des répliques avec extraction.
\end{abstract}

Abstract. - The chemical analysis of a very small area of a specimen requires simultaneous observation of this specimen at a convenient magnification. On the electron microscopy scale, two methods have been applied with thin film specimens :

- Spectroscopy of transmitted electrons : the energy losses of these electrons give a characteristic spectrum, which depends on the nature of the absorbing element or compound. For this purpose a velocity filter has to be attached to a conventional electron microscope.

- X-emission microanalysis, in a combined electron microscope-electron probe microanalyser.

In the first case, the resolving power may be as good as that of the conventional electron microscope ; in the second case, resolution of about 2000 to $3000 \AA$ has already been achieved but values of $200 \AA$ should be possible. However the analytical possibilities of $\mathrm{X}$ ray microanalysis are certainly much greater, because the $\mathrm{X}$ spectra are much finer and more selective than the energy loss spectra.

For metallurgical applications, the specimens can be thin films or extraction replicas.

I. Introduction. - Les renseignements fournis par la microscopie électronique complétée par la microdiffraction électronique sont parfois insuffisants pour le métallurgiste. Celui-ci s'intéresse non seulement au faciès microscopique, aux défauts de structure, à l'identification de la nature cristallographique des phases constitutives, mais encore à leur composition chimique. Fréquemment d'ailleurs, l'identification d'un précipité à l'aide de son diagramme de diffraction n'est pas univoque, et l'analyse chimique vient apporter une information supplémentaire utile (cas de composés isomorphes par exemple). Le problème posé est donc celui de l'analyse élémentaire très localisée. Le microanalyseur à sonde électronique ou " microsonde » maintenant largement répandu dans les laboratoires ne répond pas directement à ce besoin, puisque son pouvoir de résolution est limité aux environs du micron [1], [2].
Une analyse très localisée n'est possible que si un signal caractéristique de la nature des atomes présents peut être détecté avec une intensité suffisante, ou plutôt si le rapport signal/bruit est assez élevé pour que l'analyse puisse être effectuée avec le pouvoir de résolution requis dans un temps raisonnable. Bien que la mesure de la densité locale ait été préconisée (Marton et Schiff, 1941) [3], les variations d'épaisseur des objets rendent peu praticable cette méthode. Reste donc, puisque nous ne traiterons pas ici la microdiffraction, l'analyse chimique locale. Celle-ci peut être fondée sur l'étude des électrons rétrodiffusés, des électrons transmis ou du rayonnement $\mathrm{X}$ émis. Les caractéristiques des électrons rétrodiffusés (rendement total et distribution énergétique ou angulaire de cette émission) pourraient peut-être permettre certaines analyses, bien que l'effet de l'épaisseur variable ou inconnue de l'objet, préparé sous forme de 
lame mince pour l'examen au microscope électronique par transmission, apporte quelques restrictions. Rappelons en effet que la rétrodiffusion est une propriété de « volume », c'est-à-dire caractéristique d'une épaisseur de l'échantillon de l'ordre de grandeur de la pénétration des électrons et qu'ainsi les propriétés des électrons rétrodiffusés dépendent de l'épaisseur de la lame. Deux autres méthodes ont été étudiées et ont conduit à des réalisations pratiques d'un grand intérêt pour le métallurgiste : elles utilisent respectivement les électrons transmis et le rayonnement $\mathrm{X}$ émis.

II. Electrons transmis. - 1 . PropriétÉs ÉNergéTIQUes. - Les électrons monocinétiques incidents subissent au cours de leur traversée de l'objet des collisions avec les électrons ou avec les noyaux. On distingue les collisions élastiques et inélastiques : au cours de ce deuxième type de processus, l'électron incident perd une certaine quantité d'énergie. Finalement, les électrons transmis présentent un spectre énergétique $n(E)$ ou, ce qui revient au même, un spectre de pertes d'énergie $\Delta E=E_{0}-E$ ( $E_{0}$, énergie incidente). Ce spectre est constitué d'un continuum d'intensité croissante avec $\Delta E$ auquel se superpose un spectre de raies. Ce dernier est caractéristique de la nature des atomes de l'objet. Voyons son origine.

2. ORIGINE DU SPEGTRE DE RAIES DE PERTES D'ÉNERGIE [4]. - Ce spectre provient des collisions inélastiques. Si pour un type de processus l'électron perd une énergie $\Delta E_{i}$, on observera donc des raies pour les pertes $\Delta E_{i}, 2 \Delta E_{i} \ldots$, d'intensité décroissante. Si plusieurs pertes $\Delta E_{i}, \Delta E_{j} \ldots$, sont possibles, on observera les diverses combinaisons linéaires de celles-ci (par suite des diffusions successives d'un même électron incident). A la limite on aura un spectre continu de pertes.

Pertes d'énergie par ionisation des atomes, ou pertes lointaines. - Ces pertes d'énergie caractéristiques sont dues à l'ionisation d'un atome sur l'un de ses niveaux profonds $K, L, M$... Les raies correspondantes ont été observées pour les éléments légers (par exemple ionisation $K$ du carbone, $\Delta E=285 \mathrm{eV}$, de l'oxygène $526 \mathrm{eV})$, pour des éléments métalliques comme l'aluminium (ionisation $K, 1,5 \mathrm{keV}$ ) ou le fer (ionisation $L$, $710 \mathrm{eV}$, ou $M, 56 \mathrm{eV}$ ). Les raies correspondantes sont cependant peu intenses et se détachent assez mal sur le spectre de pertes continu surtout pour les pertes les plus lointaines. Ces raies sont souvent assez larges et apparaissent comme des bandes qui possèdent une structure fine en relation avec la nature de la liaison chimique où est engagé l'atome.

Pertes faibles. - Outre des pertes lointaines, on observe des pertes d'énergie de faible valeur (quelques $\mathrm{eV}$ à quelques dizaines d'eV), qui peuvent avoir deux origines :

- Des transitions interbandes : les niveaux d'énergie les plus externes de l'atome étant élargis en bandes, un électron peut être excité au sommet d'une bande sur les niveaux permis non occupés. Les transitions sont donc individuelles.

- Des transitions collectives, où l'excitation se traduit par des oscillations collectives de tout le gaz d'électrons de conduction : ce sont les oscillations de plasma, que l'on peut décrire en termes de création d'un « plasmon » par l'électron incident, accompagnée d'une diffusion de celui-ci.

Le second processus semble prédominant dans un grand nombre d'éléments, par exemple $\mathrm{Be}, \mathrm{C}, \mathrm{Al}, \mathrm{Mg}$, $\mathrm{Si}, \mathrm{Ge} . . .$, et il a été bien étudié. En effet, la théorie des oscillations de plasma permet de calculer leur fréquence : celle-ci varie comme la racine carrée du nombre d'électrons par unité de volume. Elle est donc caractéristique de l'élément considéré. Pour l'aluminium, par exemple, on calcule et on mesure, $\Delta E=14,6 \mathrm{eV}$. On observe bien entendu les pertes multiples de cette perte élémentaire.

Malheureusement, à côté de ces oscillations de plasma de volume, il existe des oscillations de plasma de surface, ainsi appelées car leur fréquence dépend de la constante diélectrique de la surface. Elle est d'ailleurs plus faible que celle du plasma de volume, mais varie suivant que le métal est oxydé ou contaminé. De plus sa théorie est encore mal connue.

Finalement, le spectre de pertes peut être relativement compliqué. En outre, pour les métaux de transition, les raies sont larges et leur origine n'a pas encore

\begin{tabular}{|c|c|c|}
\hline Al & $\wedge \Lambda$ & $\begin{array}{lllll}6.5 & 14.8 & 23 & 29.5 & 45\end{array}$ \\
\hline $\mathrm{Al}_{2} \mathrm{O}_{3}$ & & 22546 \\
\hline $\mathrm{Be}$ & & 193856 \\
\hline $\mathrm{BeO}$ & & $\begin{array}{llll}5.7 & 16.5 & 28 & 57 \\
\end{array}$ \\
\hline $\mathrm{Mg}$ & 1 & $10.320 .6 \quad 32 \quad 43$ \\
\hline $\mathrm{MgO}$ & & $\begin{array}{llll}4.5 ? & 5.5 & 11.4 & 25\end{array}$ \\
\hline Sn & & $\begin{array}{lll}63 & 13 & 19.5 \\
\end{array}$ \\
\hline $\mathrm{SnO}_{2}$ & & $\begin{array}{lllll}5.5 & 12.5 & 19.5 & 35 & 63\end{array}$ \\
\hline $\mathrm{Si}$ & & $5.5 \quad 22.5 \quad 45$ \\
\hline $\mathrm{SiO}_{2}$ & & $\begin{array}{lll}12.5 & 16.2 & 24.5\end{array}$ \\
\hline $\mathrm{Ag}$ & & 3.481752534 \\
\hline $\mathrm{Au}$ & & $\begin{array}{llllllll}6.5 & 17 & 5 & 25 & 34 & 50 & 62 & ?\end{array}$ \\
\hline $\mathrm{Cu}$ & & 719.5 \\
\hline Co & & 22.963 .3 \\
\hline $\mathrm{Cr}$, & & 2654 \\
\hline $\mathrm{Fe}$ & & $23.249 \quad 62$ \\
\hline$N_{1}$ & & $\begin{array}{llll}6.5 & 12 & 22.5 & 45 \\
\end{array}$ \\
\hline $\mathrm{Sb}$ & & $\begin{array}{llll}6.5 & 18 & 24.5 & 46\end{array}$ \\
\hline$T_{1}$ & & $\begin{array}{llll}6.5 & 13 & 24 & 50\end{array}$ \\
\hline TI & & $4.7 \quad 17.4 \quad 34.5 \quad 43$ \\
\hline W & & $7 \quad 22 \quad 44 \quad 54$ \\
\hline Ge & & $16.4 \quad 33.8$ \\
\hline
\end{tabular}

FIG. 1. - Spectres de pertes d'énergie de quelques éléments et oxydes, en eV, d'après [13]. 
été identifiée avec certitude. Dans un alliage, la valeur de la perte par plasmon de volume varie avec la composition (comme la racine carrée de la densité électronique). Dans certains composés, on a repéré des pertes caractéristiques, par exemple dans l'alumine à $21,4 \mathrm{eV}$ ( fig. 1).

3. Appareillage. - L'analyse chimique fondée sur les valeurs de pertes d'énergie peut être effectuée à fine échelle soit par une méthode globale (microscopie électronique), soit par une méthode ponctuelle (sonde électronique) [16].

a) Sonde électronique. - Cette méthode avait été préconisée dès 1944 par Hillier et Baker [5], [6] qui construisirent un " microanalyseur électronique » fondé sur l'examen du spectre d'énergie des électrons émergents d'un point donné de la préparation. L'appareil ne reçut pas de développements, car les pertes par ionisation utilisées pour l'analyse élémentaire étaient d'intensité trop faible par rapport au spectre continu. La microanalyse s'est par la suite développée par l'analyse du spectre $\mathrm{X}$ caractéristique émis à la suite de ces ionisations.

b) Microscopie électronique. - Il s'agit de placer dans le microscope électronique, après l'objectif, un filtre de vitesse qui permet de ne former les images qu'avec les électrons ayant une énergie bien déterminée. Citons dans ce domaine les travaux de Watanabe et Uyeda [7] utilisant un filtre du type Möllenstedt [8], les travaux de Castaing et Henry qui ont mis au point un prisme magnétique [9], [10], [11]. Avec ces appareils, on peut obtenir des micrographies électroniques formées par les électrons n'ayant subi aucune perte d'énergie ou bien par les électrons ayant subi telle ou telle perte d'énergie. La figure 2 due à El Hili [12] montre à titre d'exemple des micrographies d'un alliage $\mathrm{AlZnMg}$ recuit $72 \mathrm{~h}$ à $175^{\circ} \mathrm{C}$ : des précipités de $\mathrm{MgZn}_{2}$ apparaissent en noir sur l'image obtenue avec la perte de l'aluminium $(14,6 \mathrm{eV})$, mais c'est l'inverse pour l'image filtrée dans la bande 20-25 eV.

Par contre, Whelan et coll. étudient le spectre de pertes d'énergie de régions choisies par microscopie électronique sans former d'images filtrées [14].

4. Possibilités de la méthode. - Le pouvoir de résolution (dimension de la plus petite région analysée) est limité par le diamètre de la sonde dans le cas de la méthode ponctuelle (environ $100 \AA$ si le très faible courant de la sonde permet encore l'analyse du spectre). Au contraire, dans la méthode globale, il peut être aussi bon que celui du microscope électronique, si le filtre utilisé n'introduit aucune aberration importante (la résolution est même meilleure dans le cas d'images en champ sombre). Les micrographies publiées ont montré un pouvoir séparateur de l'ordre de $0,01 \mu[10],[12]$. Cet avantage vaut non seulement pour l'analyse de précipités, mais encore pour l'analyse de ségrégations très localisées, comme celles qui se produisent par exemple au voisinage des joints de grains ou de précipités [14]. En effet, la valeur de la perte par plasmon varie avec la racine carrée du nombre d'électrons par unité de volume. Dans ce cas, un filtre extrêmement sélectif est évidemment nécessaire.
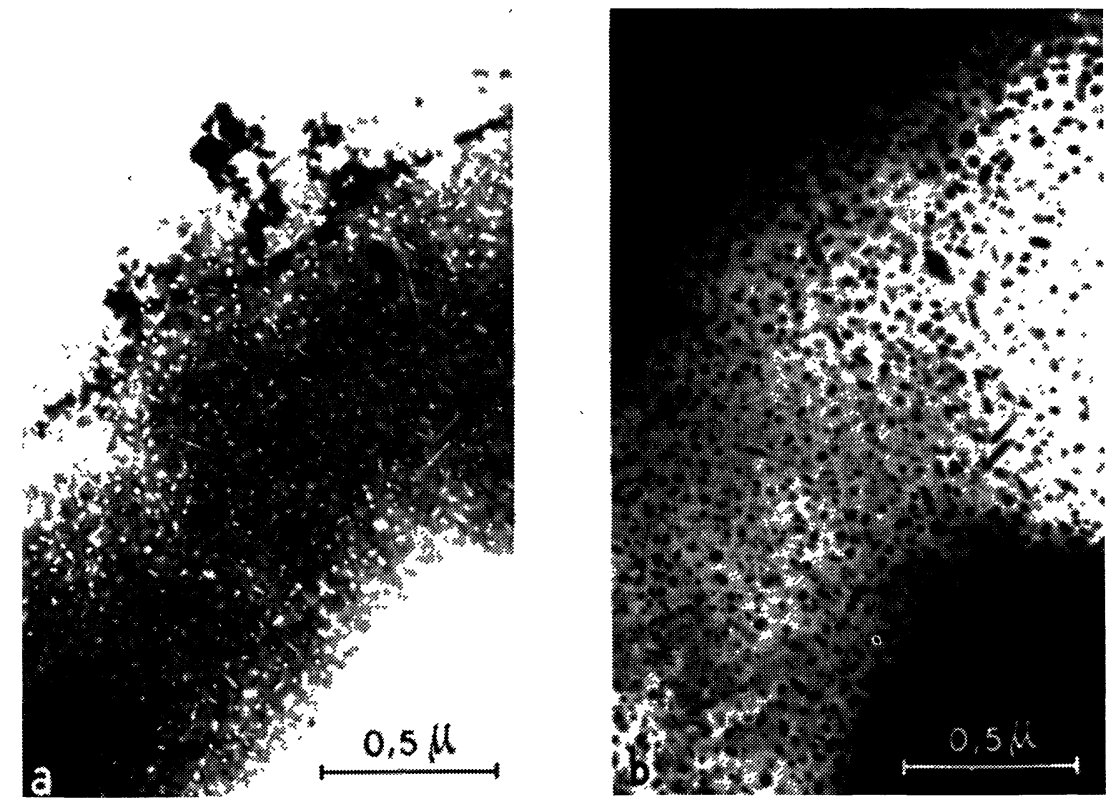

Frg. 2. - Micrographies électroniques en transmission d'un alliage $\mathrm{Al}, 7,6 \% \mathrm{Zn}, 2,6 \% \mathrm{Mg}$ sous $70 \mathrm{kV}$.

a) Image filtrée sans pertes.

b) Image filtrée $\Delta E=14,6 \pm 1 \mathrm{eV}$. 
Le filtre de vitesse de Castaing et Henry a un pouvoir séparateur en énergie de $1 \mathrm{eV}$, ce qui est suffisant avec un microscope électronique normal. Avec une source d'électrons bien monochromatique, il a été possible d'obtenir un pouvoir séparateur de $0,1 \mathrm{eV}$.

Citons brièvement les inconvénients ou les limitations de cette méthode d'analyse :

On possède encore assez peu de renseignements sur les spectres de pertes d'énergie " proches » de l'ensemble des éléments chimiques (cf. la mise au point de Gauthé [4]). Pour la plupart des éléments, il n'y a pas de données sûres.

Les raies de certains éléments (métaux de transition en particulier) sont assez larges.

Comme les spectres sont caractérisés par les pertes simples et multiples (et par les plasmons de surface), les risques de superposition sont importants. La méthode est donc peu sélective.

Le pouvoir de résolution n'est pas limité par le microscope, mais par le volume minimal nécessaire à l'excitation des oscillations de plasma. Il est d'ailleurs probable que la valeur de $\Delta E$ varie lorsque le volume mis en jeu décroît (cas de très petits précipités).

Le fond continu n'est pas négligeable et les images ne sont jamais aussi fortement contrastées que le permettrait en principe la méthode.

Il s'agit nécessairement d'analyse qualitative, éventuellement semi-quantitative dans quelques cas particuliers.

L'utilisation des pertes lointaines (ionisation des niveaux profonds) permettrait une analyse plus sûre, puisque les niveaux d'énergie sont parfaitement connus. A cette possibilité, deux limitations sont à noter :

- Le faible contraste des raies sur le spectre continu de pertes d'énergie.

- En microscopie électronique, l'aberration chromatique limite le pouvoir séparateur d'images obtenues avec des électrons ayant subi une forte perte d'énergie. Selon Watanabe [13], le pouvoir de résolution atteindrait $800 \AA$ pour une perte d'énergie de $400 \mathrm{eV}$ (électrons incidents de $50 \mathrm{keV}$ ). Ainsi la méthode pourrait en particulier s'appliquer aux éléments de faible numéro atomique (énergie d'ionisation faible, spectre dépendant de l'état de valence). Elle pourra probablement recevoir de nouveaux développements grâce aux intensificateurs de contraste ou aux tubes à mémoire.

III. Microanalyse par spectrométrie X. - 1. PRINGIPE. - Dès 1948, Castaing et Guinier montrèrent que le rayonnement $\mathrm{X}$ émis à l'impact d'une sonde électronique d'un diamètre d'environ $1 \mu$ est assez intense pour que son spectre permette l'analyse élémentaire qualitative et quantitative du microvolume bombardé. Castaing put ainsi construire le premier microanalyseur à sonde électronique [1], [2].
On peut se demander si cet appareil peut fournir des images caractéristiques de la composition chimique de l'objet. Puisque le signal émis est constitué de rayons $\mathrm{X}$, la seule méthode possible est la méthode ponctuelle, par sonde, et non la méthode globale, les rayons $\mathrm{X}$ ne pouvant pratiquement pas être utilisés directement pour former des images. C'est pourquoi des images ne peuvent être obtenues que par reconstitution point par point à partir des émissions $\mathrm{X}$ des points correspondants de l'objet. Cette méthode séquentielle est obtenue par balayage ligne par ligne de la surface de l'objet grâce à une déflexion de la sonde. Elle fut mise en œuvre pour la première fois par Cosslett et Duncumb [15].

2. Pouvoir de RÉsolution. - La microanalyse par émission $\mathrm{X}$ est-elle possible à l'échelle du microscope électronique, c'est-à-dire à une échelle beaucoup plus fine que le micron? On sait en effet former des sondes électroniques dont le diamètre est d'une centaine d'angströms. Or, en fait, le pouvoir de résolution n'est pas limité par le diamètre de la sonde, mais par la pénétration diffuse des électrons dans l'objet, de sorte que la dimension du microvolume analysé dépend essentiellement de l'énergie des électrons incidents, énergie qui elle-même doit être assez élevée pour exciter le rayonnement $\mathrm{X}$ des éléments à analyser (5 à $35 \mathrm{kV}$ généralement). Le pouvoir de résolution est donc limité nécessairement à 1 ou $2 \mu$.

Cette première limite peut être levée en utilisant comme objets non plus des échantillons massifs mais des échantillons minces, plus précisément suffisamment minces pour que la diffusion des électrons y soit pratiquement négligeable. Les trajectoires des électrons dans l'objet sont alors quasiment rectilignes.

Dans ces conditions, le pouvoir de résolution est considérablement amélioré par rapport à celui de la microanalyse sur objets massifs - sans pouvoir atteindre celui du microscope électronique par transmission pour les raisons qui ont été exposées à propos du microscope électronique à balayage : le diamètre minimal de la sonde se situe aux environs de $50 \AA$ [16].

D'autre part, contrairement à ce dernier instrument et l'effet de matrice étant éliminé, il est ici intéressant d'utiliser des tensions d'accélération les plus élevées possible, pour trois raisons principales :

- La section efficace de diffusion des électrons diminue quand leur énergie augmente. La partie quasi rectiligne de la trajectoire est donc accrue, ce qui permet d'utiliser des échantillons plus épais.

- Le rapport signal/bruit, c'est-à-dire l'intensité des raies caractéristiques par rapport au fond continu, augmente avec l'énergie excitatrice.

- La brillance du canon - donc de la sonde augmente avec la haute tension, d'où des courants transportés plus intenses pour une même dimension de la sonde.

Mais une seconde limite est liée à la nécessité d'avoir un rapport signal/bruit assez élevé qui permette une 
analyse à la précision demandée dans un temps raisonnable. Cette condition implique une certaine valeur du courant de la sonde, valeur qui dépend du rendement de l'émission $\mathrm{X}$ et $\mathrm{du}$ spectromètre pour l'élément et la longueur d'onde utilisés. Mais on sait que le courant maximal transporté par une sonde varie comme la puissance $8 / 3$ de son diamètre (à cause de l'aberration de sphéricité de la lentille qui focalise la sonde). Le diamètre de la sonde est donc limité par cette condition, que l'on rencontre également en microscopie électronique par balayage; mais ici elle est beaucoup plus restrictive; en effet, le signal est beaucoup moins intense (alors que le nombre d'électrons secondaires émis est de l'ordre de grandeur du nombre d'électrons incidents, ce rapport tombe à des valeurs $10^{4}$ ou $10^{5}$ fois plus faibles par les rayons $\mathrm{X}$ ) et le bruit de fond est plus important (présence d'un spectre X continu). En supposant les meilleures conditions réunies (brillance de la sonde, aberration des lentilles, rendement du spectromètre), Castaing a situé vers $200 \AA$ le pouvoir de résolution ultime de la microanalyse à sonde électronique par émission $\mathrm{X}$ [2]. Il est probable qu'une telle résolution puisse être atteinte sur des échantillons métalliques minces. Elle serait probablement moins bonne avec des objets biologiques, l'échauffement de ceux-ci limitant les valeurs du courant sonde.

3. Appareils. - L'analyse à l'échelle considérée n'offre d'intérêt que si l'échantillon peut être examiné à la même échelle, en particulier afin de choisir les points à analyser d'après leur faciès micrographique ou leur diagramme de diffraction. En bref, il s'agit essentiellement de combiner microscopie électronique et microanalyse à sonde électronique. Aussi du point de vue appareillage trois voies se sont-elles ouvertes :

- Partir d'un microscope électronique et lui adjoindre soit un ou plusieurs détecteurs de rayons $\mathrm{X}$ (spectrométrie non dispersive), soit un ou plusieurs spectromètres à cristal. Ceci a été fait par divers constructeurs avec une perte du pouvoir de résolution du microscope, l'objet devant être déplacé pour permettre la collecte des rayons $\mathrm{X}$ avec une dimension de la sonde d'environ $1 \mu$ ou plus - et sans offrir les diverses « facilités » des microanalyseurs (possibilité de balayage, de collecte des électrons secondaires, ou du courant échantillon, etc.) [17], [18].

- Partir d'un microanalyseur et lui adjoindre un microscope électronique. C'est ainsi qu'un constructeur a conçu un « module » de microscopie électronique qui s'échange très facilement avec le compartiment objet classique [19]. Ce microscope est équipé de deux lentilles, il offre un pouvoir de résolution meilleur que $200 \AA$, ce qui permet l'observation à un grossissement atteignant 10000 fois (fig. 3).

La haute tension est malheureusement limitée à $35 \mathrm{kV}$ et la microdiffraction n'y est pas possible. Par contre, il est très facile de passer de la microscopie

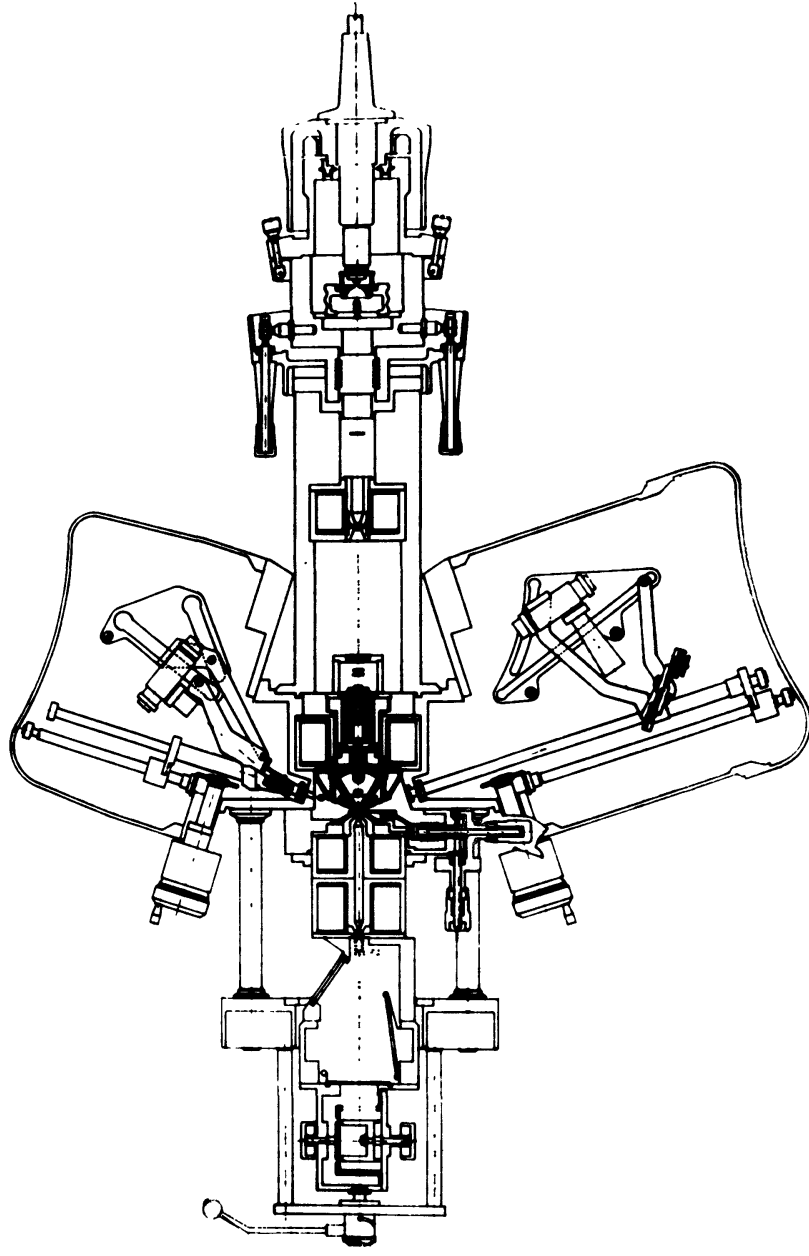

Fig. 3. - Schéma de l'ensemble microanalyseur à sonde électronique + microscope électronique construit par Cameca.

électronique à la microanalyse par sonde électronique en agissant sur l'excitation de l'objectif focalisateur qui sert soit de second condenseur pour éclairer l'objet, soit de lentille formant la sonde sur l'objet. L'image peut également être obtenue par balayage.

- Construire un appareil spécialement étudié. Citons à cet égard l'appareil à deux canons de Nixon [20] et l'E.M.M.A. (Electron Microscope and Micro-Analyser) de Duncumb [21], [22]. La figure 4 donne un schéma de cet instrument. La partie inférieure permet de focaliser une sonde de 0,1 à $0,2 \mu$ de diamètre, et l'ensemble constitue un microscope électronique dont la résolution est d'environ $100 \AA$. L'appareil peut fonctionner de 5 à $50 \mathrm{kV}$. E.M.M.A. permet :

- la microscopie électronique en transmission,

- la microscopie électronique par balayage par électrons secondaires ou rétrodiffusés, 
- la microscopie électronique par balayage par électrons transmis,

- la microdiffraction électronique,

- la microanalyse par émission X (dispersive et non dispersive).

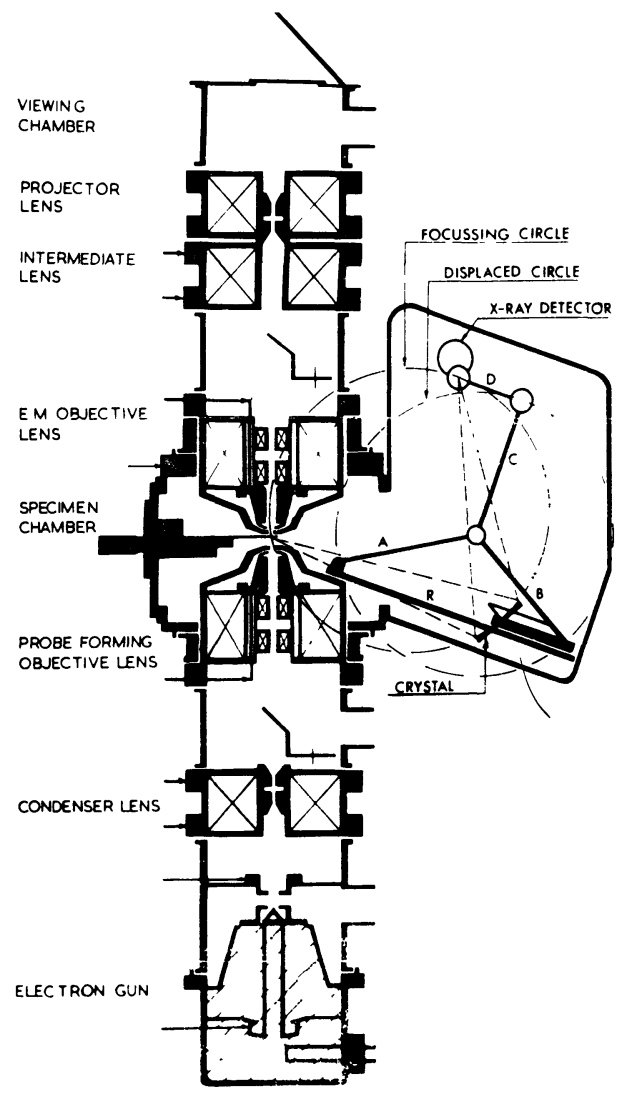

FIG. 4. - Schéma de E.M.M.A. I (Electron Microscope Micro-Analyser) de P. Duncumb [21].

4. Possibilités ex limites. - L'intérêt essentiel réside dans l'amélioration du pouvoir séparateur. Nous avons vu que celui-ci pourrait atteindre $200 \AA$, mais on n'en est pas encore là à l'heure actuelle. En fait, pour de nombreuses analyses, on est limité par la faible intensité du rayonnement $\mathrm{X}$ émis, par suite de la faible valeur du courant sonde $\left(\sim 10^{-9} \mathrm{~A}\right)$ nécessaire à l'obtention d'une sonde de 0,1 à $0,2 \mu$ de diamètre et par suite des très petits volumes de matière analysés. Ces limitations conduisent à mettre en œuvre dans certains cas les techniques de spectrométrie non dispersive à grand rendement de collecte des photons $\mathrm{X}$, méthodes moins souples et moins précises que la spectrométrie à cristal.

Une autre limitation actuelle vient de la valeur maximale de la haute tension $(\leqslant 50 \mathrm{kV})$, ce qui limite beaucoup l'épaisseur maximale des objets analysables et observables et réduit l'intérêt de la méthode aux yeux des spécialistes de microscopie électronique.

Enfin, les possibilités d'analyse quantitative sont limitées, parce que la masse de matière excitée n'est pas connue. On doit alors comparer les émissions des éléments pris deux à deux; cette analyse est possible si l'on dispose de plusieurs spectromètres permettant l'analyse simultanée de ces éléments au même point. Mais les diverses corrections à apporter aux intensités mesurées sont encore loin d'être au point [23] comparativement à la situation actuelle pour l'analyse habituelle d'objets massifs.

5. Applications métallurgiques. - Malgré le très grand intérêt de cette méthode combinée pour la biologie, nous nous limiterons ici à une évocation des applications métallurgiques.

Deux types d'objets peuvent être utilisés :

- les lames minces,

- les répliques avec extraction.

Du fait des faibles valeurs de la haute tension dans les appareils actuels, ce sont surtout les répliques avec extraction qui ont été utilisées. Elles offrent d'ailleurs un second avantage, qui est d'analyser des précipités extraits plus petits que le diamètre de la sonde, à condition que ceux-ci soient suffisamment isolés sur la réplique : le pouvoir de résolution pratique peut être alors beaucoup plus petit que le diamètre de la sonde. Des précipités en forme de plaquettes de moins
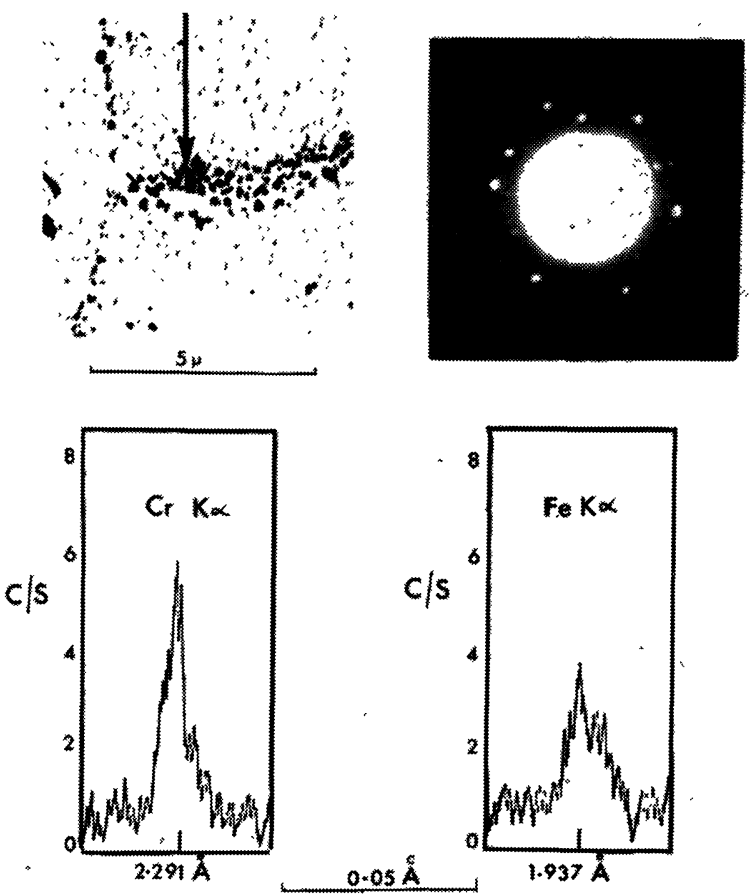

FIG. 5. - Application d'E.M.M.A. à l'identification et l'analyse de précipités de carbures de chrome extraits d'un acier inoxydable : successivement micrographie électronique montrant la particule choisie, diagramme de diffraction de cette particule, spectrogrammes des raies $\mathrm{K} \alpha \mathrm{Cr}$ et $\mathrm{K} \alpha \mathrm{Fe}$ de la même particule. D’après [22]. 

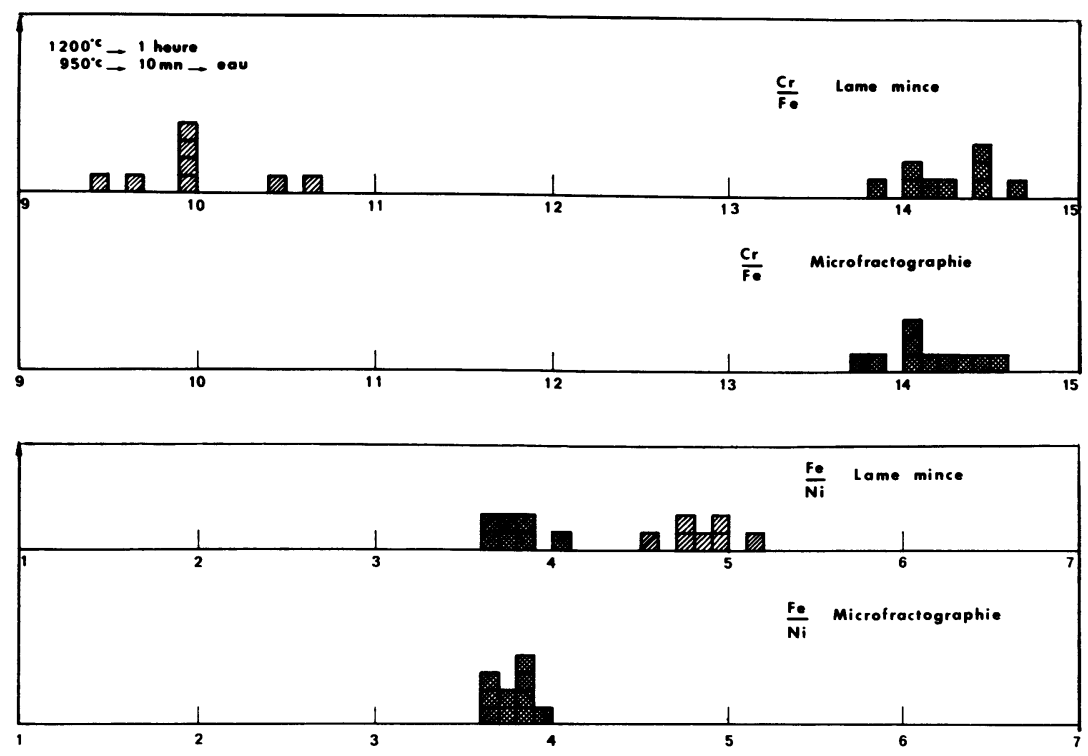

FIG. 6. - Analyse de précipités extraits sur réplique.

Comparaison des histogrammes des rapports de concentration obtenus avec des extractions à partir d'une lame mince et d'une surface de rupture.

de $500 \AA$ de côté et d'épaisseur bien inférieure ont ainsi pu être analysés.

La figure 5 montre un exemple d'analyse complète [22] : micrographie électronique en transmission, microdiffraction électronique, enregistrement des spectres $\mathrm{X}$ des éléments présents; l'identification des précipités extraits est alors assez sûre, le diagramme de diffraction et l'analyse chimique élémentaire fournissant des informations complémentaires.

La méthode peut être considérée comme fidèle. A titre d'exemple, la figure 6 montre les histogrammes des rapports $\mathrm{Fe} / \mathrm{Cr}$ et $\mathrm{Fe} / \mathrm{Ni}$ dans des carbures obtenus sur une réplique avec extraction préparée sur une section polie et attaquée d'un acier inoxydable. Cette analyse a été effectuée dans un microanalyseur classique où il n'est pas possible d'observer la réplique. L'histogramme montre clairement la présence de deux types de précipités. On peut penser que cette double distribution pourrait correspondre à la présence de précipités inter- et intragranulaires. Pour vérifier cette hypothèse, une réplique avec extraction a été préparée à partir d'une surface de cassure intergranulaire (microfractographie) obtenue par rupture à basse température. L'histogramme ne montre alors qu'un seul type de précipités dont l'analyse (rapport de $\mathrm{Fe} / \mathrm{Cr}$ ) coïncide bien avec l'une des valeurs obtenues sur les répliques précédentes.

Les possibilités d'analyse ont été étendues depuis quelques années du côté des éléments très légers, ce qui permet la détection d'éléments très intéressants pour le métallurgiste, tels que $\mathrm{B}, \mathrm{C}, \mathrm{N}, \mathrm{O}$. On a montré que ceux-ci peuvent être analysés sur répliques. Il suffit de préparer non seulement les répliques habituelles en carbone, mais aussi, pour l'analyse de cet élément, des répliques en monoxyde de silicium [24]. L'étude des précipités de composés semi-métalliques tels que carbures, nitrures, carbonitrures, borures, etc., est ainsi ouverte. Cependant, il convient d'être réservé sur la possibilité d'analyse quantitative de ces éléments dans les précipités extraits.

Notons enfin une application à la théorie du contraste en microscopie électronique. Sur des lames minces d'or, Duncumb a observé un renforcement de l'émission $\mathrm{X}$ suivant les contours d'extinction des micrographies. Cet effet est relié à la pénétration anomale des électrons au voisinage de l'incidence de Bragg [25].

Conclusion. - La microanalyse par les pertes d'énergie des électrons semble, en dépit de son intérêt, ne présenter que des possibilités limitées. Cependant, le filtre de vitesse apparaît comme un accessoire utile pour le microscope électronique classique du fait des informations complémentaires qu'il apporte pour aider à l'identification des microconstituants. En outre, la possibilité d'utilisation des pertes lointaines en complément au microanalyseur classique ou du type E.M.M.A. pourrait être à nouveau étudiée [13].

L'intérêt des appareils combinant microscopie électronique et microanalyse à sonde électronique est évident, et leur développement certain dans les années à venir. Il serait souhaitable que les constructeurs 
s'intéressent à ce type d'appareil. Le métallurgiste, dans la course aux alliages à haute résistance, est en effet confronté quotidiennement à des problèmes d'analyse et d'identification de microprécipités (composés intermétalliques et semi-métalliques). Les techniques d'extraction sont maintenant bien au point, en particulier grâce aux attaques sous potentiel contrôlé, à l'aide du potentiostat.

La possibilité d'examen de lames minces ouvrirait de nouvelles applications : particules encore plus fines, microségrégations au voisinage des précipités, des joints de grains, etc.

\section{BIBLIOGRAPHIE}

[1] Castaing (R.), Publication O.N.E.R.A., 1951, n ${ }^{0} 55$.

[2] Castaing (R.), Advances Electronics Electron Physics, 1960, 13, 317.

[3] Marton (L.) et Schiff (L. I.), J. Appl. Phys., 1941, 12, 759.

[4] GaUThÉ (B.), Les pertes d'énergie caractéristiques des électrons dans les solides, Gauthier-Villars, Paris, 1968.

[5] HILLIER (J.), Phys. Rev., 1943, 64, 318.

[6] Hillier (J.) et BAKER (R. F.), J. Appl. Phys., $1944,15,663$.

[7] Watanabe (H.) et Uyeda (R.), J. Phys. Soc. Japan, 1962, 17, 569 .

[8] MöllenstedT (G.), Optik, 1949, 5, 499.

[9] Castaing (R.) et Henry (L.), C. R. Acad. Sci., 1962, 255, 76.

[10] Henry (L.), Bull. Soc. Fran. Minéral. Crist., 1964, 88, 172.

[11] Castaing (R.), El Hili (A.) et Henry (L.), Optique des rayons $\mathrm{X}$ et microanalyse, Hermann, Paris, 1966 , p. 77.

[12] EL HILI (A.), J. Microscopie, 1966, 5, 669.

[13] Watanabe (H.), Optique des rayons $\mathrm{X}$ et microanalyse, Hermann, Paris, 1966, p. 72.
[14] Cundy (S. L.), Methereli (A. J. F.) et WheI AN (M. J.), 6th Inter. Congress Electron Microscopy, Kyoto, 1966, 1, 87; Phil. Mag., 1968, 17, 141.

[15] Cossletit (V. E.) et Duncumb (P.), Nature, 1956, 177, 1172

[16] Voir Phil.IBERT (J.), article dans ce même journal, p. 325.

[17] Fuchs (E.), Rev. Sci. Instrum., 1966, 37, 623.

[18] Giraud-HÉraud (F.), SifferLein (R.) et SaulNIER (A.), J. Microscopie, 1965, 4, 587.

[19] Rouberol (J. M.), Tong (M.), ConTy (C.) et Deschamps (P.), Microchimica Acta, 1967, Suppl. II, 201.

[20] Nixon (W. C.) et Buchanan (R.), 3rd Int. Symp. $\mathrm{X}$ Ray Optics Microanalysis, Acad. Press, New York, 1963, p. 441.

[21] Duncumb (P.), 5th Int. Conf. Electron Microscopy, Philadelphia, 1962, K K 4.

[22] Duncumb (P.), The Electron Microprobe, J. Wiley, New York, 1966, p. 490

[23] Tixier (R.) et Philibert (J.), Brit. J. Appl. Phys., 1968.

[24] Campos Soares (R.), Voeltzei, (J.) et Henry (G.), J. Microscopie, 1968, 7, 1, 17.

[25] Duncumb (P.), Phil. Mag., 1962, 7, 2101. 